\title{
Die Falle der komplementären Opferidentitäten in Großgruppenkonflikten
}

\author{
Anna Lübbe*
}

\begin{abstract}
Anwar el Sadat is credited with saying that the psychological barrier constituted 70 percent of the problem between Israel and Egypt in the 1970s. The fatal power of detrimental perceptions and constructions of reality in large group conflict is underestimated. A first step to reveal this dimension is to deconstruct its entrapping power. This article analyzes a self-organizing entrapment mechanism, using the secession conflict between Georgia and Abchazia as an example, and discusses approaches to work on such psycho-political deadlocks.
\end{abstract}

Keywords: Large group conflict, large group psychology, victim identity, dialogue projects Großgruppenkonflikt,Großgruppenpsychologie, Opferidentität, Dialogprojekte

\section{Die selbstorganisierende Beziehungsdynamik komplementärer Opferidentitäten}

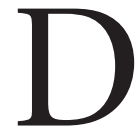
ie Sezessionskonflikte um Abchasien und Südossetien haben sich bisher als verhandlungsund mediationsresistent erwiesen (Gruska 2005; Kaufmann 2007; Halbach 2010). Sie sind mit dem Zerfall der Sowjetunion aufgebrochen und flammen seither in Form von Provokationen, Vertreibungen und Kriegen immer wieder auf. Hunderttausende sind von Flucht und Vertreibung betroffen (Gruska 2005). Die jahrelangen gegenseitigen Blockaden erschweren eine gesunde Wirtschaftsentwicklung und begünstigen soziale Disparitäten, Korruption und organisierte Kriminalität. Wertvolle Ressourcen sind in Kontrolle und Kampf gebunden, statt für nachhaltige Entwicklungen und fruchtbare Kooperationen zur Verfügung zu stehen. Die unvereinbaren Positionen in der Frage des Status von Abchasien und Südossetien drohen auch die nach der militärischen Eskalation vom August 2008 unter der Leitung der von VN, OSZE und EU begonnenen Genfer Gespräche - mittlerweile in der 15. Runde - immer wieder zu sprengen.

Die Mediationsresistenz des Konflikts hat viele Gründe. In der Konfliktforschung werden zunehmend auch psychopolitische Faktoren für die Verhärtung von Großgruppenkonflikten analysiert (Volkan 2003; 2006a; Kelman 2009; Kaufman 2001; Simon 2004; Wallach 2006; Krell 2009, S. 385 ff.; Lübbe 2009; 2010). Insbesondere geht es hier um Dynamiken, die den Charakter einer Falle haben, also die Akteure in eine ausweglose Situation führen. Paul Meerts (2005) hat Fallen in internationalen Verhandlungen analysiert. Stuart Kaufman (2006: 205) hat die „symbolic politics trap“ als ein Dilemma politischer Führer herausgearbeitet, die durch ethnisierende Propaganda an die Macht kommen und sich später durch einen Deeskalationskurs innenpolitisch in Gefahr bringen würden. Nahezu unlösbar können Blockaden werden, die sich aus einer Verschränkung des Handelns der Konfliktparteien heraus selbst organisieren:

\footnotetext{
Prof. Dr. Anna Lübbe ist Juristin, Mediatorin und systemische Beraterin. Sie lehrt und forscht an der Hochschule Fulda mit den Schwerpunkten Öffentliches Recht und Konfliktforschung. Sie supervidiert MediatorInnen systemisch und führte den systemischen Ansatz auch in die Konfliktforschung ein. Der Beitrag geht auf einen Vortrag zurück, den die Verfasserin 2010 auf einer internationalen friedenspsychologischen Tagung am Institut für Interdisziplinäre Konflikt- und Gewaltforschung der Universität Bielefeld gehalten hat.
}

Die Akteure verfolgen nach bestem Wissen ihre Interessen, im Zusammenspiel mit der anderen Partei entsteht aber eine von niemandem beabsichtigte und in diesem Sinne selbstorganisierende Dynamik, durch die die Akteure letztlich gemeinsam das Gegenteil von dem konstruieren, was sie eigentlich anstreben. Dem Sicherheitsdilemma in internationalen Beziehungen liegt ein solcher Mechanismus zugrunde. Ein für ethnopolitisierte Großgruppenkonflikte typischer, selbstorganisierender Blockademechanismus sei im Folgenden am georgisch-abchasischen Beispiel herausgearbeitet.

\subsection{Ethnopolitisierung}

Bei dem Konflikt zwischen Georgien und Abchasien handelt es sich um einen ethnopolitisierten Konflikt in dem Sinne, dass die Unterscheidung zwischen Freund und Feind entlang ethnischer Grenzen verläuft. Die Ursachen für ethnopolitisierte Konflikte sind vielfältig und einzelfallspezifisch. Ethnische Differenz allein macht jedenfalls noch keinen Konflikt. Unterschiedliche Ethnien können ohne Weiteres friedlich koexistieren und tun dies in vielen Ländern der Welt. Die Konfliktforschung hat einige Faktoren ausgemacht, die eine Eskalation von Großgruppenkonflikten entlang ethnischer Gruppengrenzen zu begünstigen scheinen (Ropers 1995; Kaufman 2001; Volkan 2003, 2006a). Dazu gehört eine schwache gesamtstaatliche Integrationskraft, typischerweise bei Staatenbildung auf tribalistischer Grundlage und unter externem Einfluss von konkurrierenden Großmächten. Diese Konstellation besteht entlang der instabilen Linie Balkan-Kaukasus-Zentralasien. Im Kaukasus kommt eine ethnisierende Wirkung der sowjetischen Nationalitätenpolitik dazu (Gruska 2005). Ein weiterer Faktor ist die Destabilisierung durch eine politische Übergangssituation, im Kaukasus durch den Zerfall der Sowjetunion. Hinzu kommt eine ethnopolitisierende Propaganda durch Großgruppenführer, deren Stunde in unsicheren und konfliktträchtigen Zeiten schlägt.

Ist der Konflikt einmal ethnopolitisiert, ist er nicht mehr wie ein Verteilungskonflikt verhandel- und vermittelbar, er hat einen existenziellen, die Identität der Parteien betreffenden Charakter bekommen. Realpolitische Analysen und Bewältigungsversuche unterschätzen den Einfluss solcher tiefer gehender, die Identität der beteiligten Kollektive betreffender Faktoren. 
Die Ethnopolitisierung ergibt sich aus einer Kollusion, also einem unbewussten Zusammenwirken, zwischen Schicksalsgemeinschaften (Ropers 1995), die in schwierigen und instabilen Zeiten zusammenrücken und empfänglich für Heilsversprechungen werden, und scheinbar starken, narzisstischen Führungsfiguren (Volkan 2006b), die diese Erwartungen bedienen - und dabei charismatischer sind, wenn sie selbst glauben, was sie den Menschen erzählen. Solche Dynamiken lassen sich nicht in Abhängigkeit von aktuellen realpolitischen Interessenlagen an- und dann wieder abschalten, sondern verselbständigen und verfestigen sich so, dass die Beteiligten nicht mehr aus ihnen herausfinden, selbst wenn die Aussichtslosigkeit und die enormen Kosten dieser Art der Interessenverfolgung längst zutage liegen (Lübbe 2009). Wie also tragen solche Identitätsaspekte im georgisch-abchasischen Fall zur Mediationsresistenz des Konflikts bei?

\subsection{Kein Übergang von den Positionen zu den Bedürfnissen}

Bestandteil gelingender Mediationen ist ein Übergang von den unvereinbaren Positionen der Konfliktparteien zu den dahinter liegenden Bedürfnissen. Der Übergang ist blockiert, wenn er sich den beteiligten Kollektiven in der ihre Politiken dominierenden Wahrnehmung als Selbstaufgabe darstellt, weil die Positionen existentiell besetzt sind. Im georgisch-abchasischen Konflikt betrifft das die Statusfrage. Die Positionen lauten auf georgischer Seite: Abchasien ist und bleibt ein Teil Georgiens, und auf abchasischer Seite: Abchasien gehört nicht und wird nie wieder zu Georgien gehören. Diese Positionen sind in der Tat unvereinbar. Eine größere Vielfalt an Optionen mit dann möglicherweise auch konsensfähigen Lösungen könnte sich allenfalls auf der Ebene der Bedürfnisse eröffnen (Sicherheit, Autonomie, gerechte Verteilung von Lasten und Ressourcen, usw.). Ist der Übergang von den Positionen zu den Bedürfnissen blockiert, bleibt die Welt, in der die Parteien leben, eine, in der es nur Sieg oder Niederlage geben kann. Um die Fixierung der Parteien auf ihre Positionen in der Statusfrage zu verstehen, muss man die mediationstypische Zukunftsorientierung verlassen und die historische Dimension eröffnen. Wie also konstruieren die Konfliktparteien vor dem Hintergrund ihrer identitätsprägenden Geschichtsbilder ihre Realität?

\subsection{Realitätskonstruktionen aus der Opfer- perspektive}

Die postsowjetische Übergangssituation hat sowohl auf der abchasischen als auch auf der georgischen Seite im Geschichtsbild der jeweiligen Großgruppe verwurzelte Opfererwartungen salient werden, also in den Vordergrund treten lassen. Im Falle Abchasiens handelt es sich um die Angst vor Auslöschung als Gruppe mit einer unterscheidbaren ethnischen Identität. Sie wurzelt in der stalinistischen Deportations- und Zwangsassimilationspolitik und in ähnlichen Erfahrungen aus dem russischen Zarenreich, als nach brutal unterdrückten Aufständen Tausende Abchasen ins Exil fliehen mussten (Kaufman 2001;
Wolleh 2006). Die ethnonationalistische Propaganda Georgiens unter dem ersten Präsidenten des unabhängigen Georgien, Swiad Gamsachurdia („Georgien den Georgiern“), war geeignet, diese Opferanteile der abchasischen Identität zu aktivieren. In der die abchasische Politik dominierenden Wahrnehmung ist das Bestehen auf der Unabhängigkeit von Georgien und auch der Widerstand gegen eine die ethnischen Mehrheitsverhältnisse verschiebende Rückkehr Hunderttausender Anfang der 1990er Jahre aus Abchasien geflüchteter ethnischer Georgier (Halbach 2010) unabdingbar für den Erhalt der abchasischen Ethnie. Tragischerweise erreicht Abchasien gerade durch die Absolutheit und Heftigkeit seiner Abwehr, dass eine Wiederholung des Traumas wahrscheinlicher wird. Je mehr eine Minderheit nicht nur ihre ethnische Eigenständigkeit, sondern die Sezession betreibt, desto eher wird sich die Mehrheit ihrerseits bedroht fühlen und zu Abwehrmaßnahmen wie Unterdrückung, Vertreibung und Zwangsassimilation veranlasst sehen. Das zeigt: Die Opferperspektive wirkt tendenziell selbsterfüllend.

Die im aktuellen Konflikt wirksame georgische Opfererwartung richtet sich gegen Russland. In der die georgische Politik dominierenden Wahrnehmung droht Georgien die Fragmentierung, weil es beginnt, sich von der russischen Dominanz zu befreien. Diese Angst wurzelt in identitätsprägenden Kapiteln des georgischen Geschichtsbildes: Viele Male in seiner Geschichte hat Georgien seine Unabhängigkeit ganz oder teilweise an umgebende Großmächte verloren: Osmanen, Perser, Russen und andere haben im Lauf der Jahrhunderte Georgien ganz oder teilweise besetzt oder dominiert. Immer wieder fand es sich den spaltenden Einflüssen konkurrierender Nachbarn ausgesetzt (Kaufman 2001). Auch die georgische Retraumatisierungserwartung ist mit dem Zerfall der Sowjetunion salient geworden, und sie hat den georgischen Ethnonationalismus befördert: Kaum hat Georgien seine Eigenstaatlichkeit wiedergewonnen und möchte seine Freiheit für eine Annäherung an den Westen nutzen, unterstützt Russland den Separatismus georgischer Gebiete. Aus dieser Perspektive sind Abchasen, Südosseten und andere ethnische Minderheiten in Georgien russische Marionetten (Kaufman 2001; Gruska 2005). Dazu passt die georgische Einordnung russischer Bemühungen in der Region als „not peacekeeping, but keeping in pieces“ (Halbach 2010). Für Georgien ist die Aufgabe seiner Position in der Statusfrage - also das Loslassen Abchasiens als völkerrechtlicher Teil Georgiens - gleichbedeutend mit seinem Zerfall. Und auch Georgien befördert die Realisierung seiner zentralen Angst durch sein Abwehrhandeln, also seinen Ethnonationalismus und seine betonte Orientierung nach Westen.

\subsection{Die Falle der komplementären Opferidenti- täten}

Das Beispiel zeigt, wie sich identitätsprägende kollektive Traumata in aktuellen Konflikten eskalierend und blockierend auswirken können: Im ethnopolitisierten Konflikt werden im Geschichtsbild der ethnischen Schicksalsgemeinschaft - in „chosen traumas“ im Sinne Vamik Volkans (2003: 73) - wurzelnde, existentielle Ängste aktiviert. Geschichtsbild deshalb, 
weil die Narrative, mit denen ethnische Schicksalsgemeinschaften ihre Identität konstruieren, mit Geschichte selektiv und mythifizierend umgehen. Geschichte wird immer wieder neu und anders erzählt, je nachdem, wer sie wann in welchem Kontext und zu welchen Zwecken erzählt (Lübbe 1989). Insofern sind kollektive Identitäten nichts ahistorisch Feststehendes, sozusagen Wesensmäßiges, sondern zeitbedingt und wandelbar. In ethnopolitisierten Zeiten kommt es zu einem unbewussten „Zeit-Kollaps“ (Volkan 2004: 73): In der Wahrnehmung kann zwischen vergangenen, im Narrativ der Gruppe als Traumakapitel verbuchten Erfahrungen und dem gegenwärtigen Konflikt nicht mehr angemessen unterschieden werden. Durch diese Verknüpfung erscheinen bestimmte Positionen als existentiell unverzichtbar und ihre Aufgabe als Selbstaufgabe.

Wenn sich in einem Konflikt zwei Parteien mit erstens unvereinbaren und zweitens derart existenziell belegten Positionen treffen, ist der Konflikt blockiert. Der mediationstypische Übergang zur ergebnisoffeneren Ebene der Bedürfnisse und des kooperativen Suchens nach kreativen Lösungen findet nicht statt. Die Beteiligten stecken in der Falle der komplementären Opferidentitäten. Das ist ein tragisches Zusammenwirken historisch radizierter, selbsterfüllend wirkender Sichtweisen der Parteien, durch das sie eine gemeinsame Welt des existentiellen Kampfes konstruieren. Opferidentität heißt dabei, dass jede Partei unbewusst von salienten traumatischen Kapiteln ihres kollektiven Geschichtsbildes besetzt ist. Die Opferidentitäten sind komplementär, weil das Abwehrhandeln jeder Partei exakt die spezifische Existenzangst der jeweils anderen Partei auslöst und verstärkt: Georgiens Ethnonationalismus löst die abchasische Angst vor Auslöschung als eigenständige Ethnie aus, und Abchasiens Separatismus ruft aufs Neue Georgiens Angst vor Fragmentierung hervor. Das Zusammenwirken der beiden Opferidentitäten ist, systemtheoretisch gesprochen, ein kreuzkatalytischer Zyklus: Die aus der Opferperspektive resultierenden Verhaltensweisen der beteiligten Kollektive verstärken sich gegenseitig. Eine Falle schließlich ist das Ganze, weil die Parteien letztlich genau das kollusiv, also unbewusst abgestimmt, konstruieren, was sie zu vermeiden suchen: eine existentielle Bedrohungslage, aus der, wenn sie einmal etabliert ist, kaum noch ein Weg herausführt. Allenfalls kann der Konflikt längere Zeit auf Eis liegen. Das ist dann der Attraktor, also der stabile Systemzustand, bei dem das dynamische Konfliktsystem komplementärer Opferidentitäten unter der Rahmenbedingung hinreichender Waffengleichheit schließlich landet: ein auf Eis liegendes Pulverfass - eine Metapher, die für den Kaukasus häufig verwendet wird.

Falle der komplementären Opferidentitäten - das ist nicht einfach ein anderer Name für Sicherheitsdilemma. Freilich gibt es eine Parallele in der gegenseitigen Verstärkung der beiderseitigen Ängste und Abwehrverhalten. Der Mechanismus wird hier aber nicht neorealistisch als Begleiterscheinung einer Konkurrenz rationaler Akteure in einem Selbsthilfe-System verstanden. Es ist vielmehr eine Dynamik, die zwischen Großgruppen ausgelöst werden kann, wenn für die jeweiligen Konfliktparteien spezifische und gewissermaßen wie Schlüssel und Schloss zueinander passende Empfindlichkeiten aktiviert werden. Die Untersuchungen von Gilovitch (1981), der gezeigt hat, dass politische Urteile von unbewussten historischen
Analogien abhängen, unterstützten das Konzept, dass es hier auch um historisch radizierte Empfindlichkeiten geht. Mit der Rede von „spezifischen Empfindlichkeiten“ von Großgruppen sei aber nicht eine ethnisierende Ancient-Hatreds-These vertreten, wonach Konflikte zwischen ethnischen Gruppen auf unvordenklichen Feindschaften oder gar wesensmäßigen Unverträglichkeiten beruhen. Ich sehe, wie dargelegt, Großgruppenidentitäten als ein Konstrukt an. Sie sind wandelbar in Zeit und Kontext, aber nicht zufällig und nicht beliebig. Manche Konstrukte haben ein hartnäckiges Leben und sind leichter aufrufbar und dann auch leichter instrumentalisierbar als andere.

\subsection{Internationale Faktoren: Ein Stellvertreter- konflikt?}

Der Konflikt und seine Hartnäckigkeit erklären sich nicht nur aus dem Verhältnis zwischen Abchasien und Georgien. Internationale Faktoren, insbesondere die russische und amerikanische Konkurrenz im Südkaukasus vor dem Hintergrund von hegemonialen und Energieinteressen (Jobelius/Krumm 2009; Kaufmann 2007) kommen hinzu. Abchasiens Haltung wäre nicht so kompromisslos, wenn es sich der russischen Unterstützung nicht sicher sein könnte. Und Georgiens Präsident Micheil Saakaschwili rechnete angesichts seiner Allianz mit dem Westen im August 2008 wohl nicht mit einer derart harschen militärischen Reaktion Russlands. Der Westen hat in den August-Krieg nicht eingegriffen, finanzielle Unterstützung und militärische Ausbildungshilfen für Georgien gingen aber dem Konflikt voraus (Halbach 2010). Die konkurrierenden Großmächte haben zur Verfestigung der kompromisslosen Haltung der unmittelbar betroffenen Parteien beigetragen, indem ihre Unterstützung auf beiden Seiten Hoffnungen auf eine Durchsetzung der jeweiligen Maximalforderungen unterhielten. Und die zwischen Ost und West gespaltene Interessenlage torpediert auch die internationalen Vermittlungsbemühungen (Gruska 2005; Kaufmann 2007).

Auch hier macht sich ein den Konflikt stabilisierender, psychopolitischer Faktor bemerkbar: Indem Georgien und Abchasien sich jeweils an Verbündete anlehnen, verschärft sich der Gegensatz zwischen ihnen, während die eigentlich auch vorhandenen Gegensätze zum Verbündeten, etwa der Wunsch nach einer Vermeidung erneuter Abhängigkeiten, verblassen - eine Form der Triangulation (Simon 2010). Besonders deutlich wird das im Verhältnis zu Russland: Dass die Abchasen Russland gegenwärtig als Schutzmacht benötigen, verhindert eine angemessene Aufarbeitung der konfliktrelevanten Traumata der Stalinzeit. In der (triangulierten) Wahrnehmung der Abchasen sind insoweit allein die Georgier die Täter, während Georgien sich seinerseits als Opfer wahrnimmt (Wolleh 2006). Die Konzentration auf Georgien als Täter erspart es den Abchasen, die historischen Täteranteile Russlands in den Blick zu nehmen, von dem sie in der gegenwärtigen Situation abhängig sind und, wider Willen, zunehmend abhängiger werden. Nach allem empfiehlt es sich nicht, den Konflikt als Stellvertreterkrieg anzusehen. Die Wahrnehmung, lediglich Spielball konkurrierender Großmächte zu sein, ist selbst eine Opferperspektive, 
die eigene Verantwortungsanteile und Handlungsmöglichkeiten ausblendet. Die Anlehnung an Großmächte erspart die eigenverantwortliche Verständigung mit dem Konfliktpartner, erzeugt erneute Abhängigkeiten und lässt die Ermächtigungspotenziale regionaler Kooperationen, also auch mit Armenien und Aserbaidschan (Galtung/Jacobsen/Brand-Jacobsen 2002, S. 266-270), ungenutzt.

\section{Ansätze zur Mobilisierung psychopolitischer Blockaden}

Vorweg sei klargestellt, dass die Opferidentitätsfalle hier nicht als die Ursache für den Konflikt oder für seine Hartnäckigkeit angesehen wird. Solche verfestigten Konfliktsysteme sind durch zirkuläre Kausalitäten mit zahlreichen, sich gegenseitig stabilisierenden Faktoren und Subsystemen gekennzeichnet (Coleman 2009; Wils et al. 2006). Insbesondere stellen sich in lang andauernden Konfliktlagen auch die Subsysteme Arbeit und Wirtschaft auf die Spaltung zwischen den Konfliktparteien ein, und in Medien, Bildung und Wissenschaft verfestigen sich einseitige Darstellungen, zumal, aber nicht nur, unter Bedingungen der Isolation und eingeschränkter Meinungs-, Wissenschafts- und Medienfreiheiten (Halbach 2010; Kaufmann 2007). Will man den komplexen Interdependenzen gerecht werden, muss auf mehreren Ebenen angesetzt und geduldig der Boden für stabile Veränderungen bereitet werden, also langfristig, auf allen Ebenen der Gesellschaft und mit vielfältigen Ansatzpunkten und Methoden (Diamond/McDonald 1996; Ropers 1997). Prekäre Realitätskonstruktionen, wie die beschriebenen, für ressourcenvollere Alternativen zu öffnen, ist ein Ansatz.

\subsection{Dialogprojekte}

In der Regel finden Versuche, an dem zu arbeiten, was in konflikthaften Großgruppenbeziehungen unbewusst wirksam ist, in Dialogprojekten auf der Grassroot-Ebene statt (Ropers 2004). Als ein Beispiel für ein Dialogprojekt, das explizit kollektiv-traumatische Vergangenheit bearbeitet, seien Dan Bar Ons „To Reflect and Trust“-Gruppen genannt. Bar On brachte Nachkommen von Holocaust-Opfern und Nachkommen von Holocaust-Tätern in Story-Telling-Projekten zusammen. Um die Verbindung zu heutigen politischen Folgen herzustellen, integrierte er später auch palästinensische Jugendliche in diese Dialogprojekte (Bar On 2008). Vamik Volkan wandte sein großgruppenpsychologisches „Baum-Modell“ unter anderem auf Konflikte zwischen Esten und Russen in Estland an. Mit einem interdisziplinären Team bemühte er sich um eine Identifizierung der im Konflikt unbewusst wirksamen, zentralen Ängste der Beteiligten (Wurzeln), bearbeitete die entsprechenden Aspekte der Großgruppenidentität in Dialogprojekten (Stamm) und unterstützte den Transfer in die betreffenden Kollektive, indem er die Teilnehmer gemeinsam ein Projekt erarbeiten ließ, durch das die Großgruppenverständigung vor Ort weiterverfolgt werden konnte (Früchte) (Volkan 2004).

Letzterer Aspekt, der Transfer in die betreffenden Kollektive, ist eine Schwierigkeit, die sich in Dialogprojekten stets stellt. Es geht dabei um die Frage, wie man nicht nur Einstellungsänderungen bei den jeweiligen Dialogprojekt-Teilnehmern, sondern eine nachhaltige Breitenwirkung in den konfliktbetroffenen Gesellschaften erreicht. Die Transfermöglichkeiten hängen außer von der Stabilität der Einstellungsänderungen der Projektteilnehmer insbesondere von deren Handlungsmöglichkeiten in ihren jeweiligen Kollektiven ab. Ein nahe liegender Ansatz für die Erzielung einer großgruppenkonfliktrelevanten Breitenwirkung ist deshalb die Arbeit mit möglichst einschlägig einflussreichen Teilnehmern.

\subsection{Makropolitische Ansätze}

Die direktesten Handlungsmöglichkeiten haben regelmäßig die politischen Führungseliten. Diese sind nun allerdings für jegliche Art von Arbeit, die nicht strategisch, sondern dialogisch orientiert ist, schwierig zu erreichen. „Strategisch“ meint auf die Durchsetzung von vornherein festliegender und auch fest bleibender Agenden ausgerichtet, während „dialogisch“ eine suchende Haltung bezeichnet, die offen ist für eine mit Einstellungsänderungen einhergehende Veränderung von Agenden im Lauf der Auseinandersetzung. Politiker und Politikerinnen stehen unter Druck, zeitnah Ergebnisse zu präsentieren und dabei Positionen durchzusetzen, auf die sie sich vorab öffentlich festgelegt haben. Jedes Abrücken erscheint als Schwäche, die ihnen auch innenpolitisch gefährlich werden kann. Sie sind trainiert und gewohnt, sich bei Verhandlungen nur in ihren Funktionen zu zeigen, sich über Positionen auszutauschen, sich auf Medienwirksamkeit auszurichten und jedenfalls Gesichtsverluste zu vermeiden. Psychopolitisches Neuland zu erorbern braucht demgegenüber Offenheit für die Begleiterscheinungen von Selbst-, Fremd- und Weltbildveränderungen wie etwa Betroffenheit, Ratlosigkeit oder Trauer. Die Berichte aus Dan Bar Ons Workshops (Bar On 2008) zeigen beeindruckend, wie die teilnehmenden Jugendlichen in Phasen von Verleugnung und Verwirrung gerieten, bevor sie nach und nach immerhin akzeptieren konnten, dass auch die Gegenseite wirklich leidet.

Ein Ansatz auf der Makro-Ebene, der in Reaktion auf diese Probleme entwickelt und auch praktiziert wird, ist die sogenannte Second- oder 1.5-Track-Diplomatie (Burton 1990; Fisher 2005; Kelman 2009). Das sind informelle Beratungs- oder Dialogprojekte, typischerweise mit Teilnehmern aus Nichtregierungsorganisationen und Wissenschaft sowie Personen aus dem Umfeld politischer Entscheidungsträger. Diese Personen, oft selbst ehemalige Funktionsträger, werden darin unterstützt, gewonnene Einsichten an geeigneter Stelle in die Makropolitik einfließen zu lassen. Gegenüber offiziellen diplomatischen Begegnungen ist in informellen Workshops eine tiefer gehende Beziehungsarbeit - d.h. eine Arbeit, die den grundlegenden Ängsten und Bedürfnissen der Konfliktparteien gerecht zu werden versucht -, eher möglich. Nach Stuart Kaufman (2006) sollten makropolitische Deeskalationsansätze insbesondere durch Medienarbeit ergänzt werden. Über die Medien lassen sich für die Großgruppenidentität bedeutsame Symboliken breitenwirksam transportieren. Großgruppenführer machen sich dies in der ethnopolitisierenden Propaganda zunutze. Nach Kaufman sollte der Effekt umgekehrt genutzt werden, 
um Großgruppenführern, die sich zu einer Deeskalationspolitik bereit finden, aber befürchten, sich dadurch innenpolitisch in Gefahr zu bringen, eine breite Unterstützung innerhalb des betreffenden Kollektivs zu sichern.

Ansätze zu Dialogprojekten auf der Makrobene gibt es und gab es auch bereits im Kaukasus. Genannt sei für den abchasischen Fall der Stadtschlaining-Prozess, eine im Jahr 2000 begonnene Serie von informellen Workshops mit georgischen und abchasischen Teilnehmern, die vom Berghof Zentrum Berlin und von Conciliation Resources (London) organisiert und moderiert wurde (Wolleh 2006). Unter den in der Zusammensetzung wechselnden Teilnehmern waren Politiker, Parlamentsmitglieder, hochrangige Mitglieder der Exekutive und zivilgesellschaftliche Akteure. Die abchasische Seite bewertete das Projekt als politisch wesentlich bedeutsamer als die georgische. Intendiert war, die Parteien miteinander in Kontakt zu bringen, gegenseitiges Verständnis zu entwickeln, ausgewählte Themen gemeinsam zu vertiefen sowie spekulativ Szenarien und gemeinsame Aktivitäten zur Verbesserung der georgischabchasischen Beziehungen zu entwickeln. In den Workshops wurde deutlich, wie grundlegend sich die Sichtweisen, Annahmen und Interpretationsmuster der Parteien unterscheiden und wie tief der Vertrauensverlust insbesondere der Abchasier gegenüber den Georgiern geht. Eine gegenseitige Annäherung und mehr innerparteilicher Pluralismus konnten erreicht werden, zur Phase der Entwicklung gemeinsamer Aktivitäten kam es aber nicht (Wolleh 2006). Mit der Kodori-Krise 2006 fand der Prozess ein vorzeitiges Ende. Solche Projekte erfordern einen langen Atem, und eine entsprechend langfristige Finanzierung. Für ein neues Projekt muss sich im Kaukasus nach der Eskalation vom August 2008 wohl erst wieder ein günstigeres Klima entwickeln..

\subsection{Unterstützung zivilgesellschaftlicher Multi- plikatorinnen und Multiplikatoren}

Gerade Krisenzeiten bringen in Kollektiven oft Persönlichkeiten an die Spitze, die für dialogisches Arbeiten besonders unzugänglich sind, während gleichzeitig große Teile der Bevölkerung in zunehmendem Bewusstsein der hohen Kosten einer fortdauernden Konfrontation durchaus verständigungsorientiert sein können. Ich habe Gespräche zur Kaukasuskrise begleitet, die von der INGO-Konferenz des Europarats - der zivilgesellschaftlichen Säule des Europarats - organisiert wurden. Die Teilnehmer stammten hauptsächlich aus Nichtregierungsorganisationen und Think Tanks der vom Konflikt betroffenen Regionen, einschließlich Russlands. Die erste dieser Begegnungen fand im Dezember 2008 statt. Es war beeindruckend, wie offen und konstruktiv die Gespräche verliefen. Die Teilnehmer, und besonders die Teilnehmerinnen, zeigten ihre Betroffenheit und ihre Bedürfnisse, und der enorme Schmerz, den das Trauma des Krieges hinterlassen hat, stand bei zahlreichen Äußerungen aller Seiten deutlich im Raum. Dadurch wurde jenseits aller Freund-Feind-Dichotomien eine Basis für ein gemeinsames Menschsein spürbar. Gerade einmal vier Monate nach einer derart traumatisierenden Auseinandersetzung ist das keine Selbstverständlichkeit, selbst wenn man in Betracht zieht, dass die Begegnung nicht von der Notwendigkeit belastet war, mit konkreten Ergebnissen die makropolitischen Interessengegensätze zu überbrücken.

Im weiteren Verlauf der Gespräche wurde deutlich, dass auf dieser zivilgesellschaftlichen Ebene eine hohe Kooperationsbereitschaft und auch praktischer Ideenreichtum herrschen, gleichzeitig aber Frustration wegen der Aussichtslosigkeit, damit die Führungsebenen zu erreichen, und zwar besonders die autoritäre Führungselite Georgiens. Die georgische Führungsebene ist, auch zur innenpolitischen Stabilisierung ihrer eigenen Position, ganz auf die angeblichen Notwendigkeiten der internationalen Politik und die Statusfrage konzentriert; den Bedürfnissen der Bevölkerung und der drängenden Frage, wie Georgien zu einem Land werden kann, in dem sich ethnische Minderheiten aufgehoben statt bedroht fühlen, wird nicht ausreichend Aufmerksamkeit geschenkt. Auch dieses prekäre Verhältnis von Führung und Zivilgesellschaft (Kaufmann 2007) ist ein den Konflikt stabilisierender Faktor.

Soweit und solange also die politischen Führungseliten für dialogisch orientierte Verhandlungs- und Vermittlungsbemühungen nicht erreichbar sind, bietet sich als weiterer Ansatz die Unterstützung zivilgesellschaftlicher Multiplikatoren und Multiplikatorinnen an, also von Medienleuten, Personen aus Bildungswesen, Kunst und Wissenschaft, religiösen Autoritäten, Angehörigen von Nichtregierungsorganisationen, Parteien, Gewerkschaften, Stiftungen usw. Marco de Carvalho und Jörgen Klußmann (2010) haben in Afghanistan in von der Friedrich-Ebert-Stiftung finanzierten Seminaren großgruppenkonfliktbezogene Anliegen dieser Zielgruppe systemisch bearbeitet (zum systemischen Ansatz näher Lübbe 2010; Wils et al. 2006). In Gebieten mit chronifizierten Großgruppenkonflikten, die durch schwache oder autoritäre Staatlichkeit, Ethnisierung, Gewaltökonomien und multiple soziale Probleme gekennzeichnet sind, kann viel Transformationspotenzial im Engagement zivilgesellschaftlicher Kräfte liegen, die in der Gesellschaft meinungs- und einstellungsbildend wirken können. Ungeachtet der Grenzen und Schwächen, die zivilgesellschaftliches Engagement in der Konflikttransformation auch hat (Fischer 2006), sind zivilgesellschaftliche Institutionen mit ihrem engeren Kontakt zur Bevölkerung, ihrem oft auch kooperativen Verhältnis zu ähnlichen Initiativen auf Seiten der anderen Konfliktpartei und ihrem höheren Anteil an Frauen eine innersystemische Ressource, die zu unterstützen sich lohnt. Wesentlich ist dabei, dass die Lösungen mit den Betroffenen aus dem System selbst heraus entwickelt werden, nur dann sind sie hinreichend kultursensibel und werden als eigen empfundenen. Versuche, ein System, vor allem ein fremdkulturelles, nach Maßgabe von auf externen Analysen beruhenden, mitgebrachten Konzepten instruktiv zu verändern, erzeugen Widerstände, die oft ihrerseits auf historische radizierte Empfindlichkeiten zurückgehen (Lübbe 2007).

\section{Fazit}

Die Transformation von Großgruppenkonflikten erfordert eine Integration psychopolitischer Sicht- und Herangehensweisen in die Friedensarbeit. Letztlich verlangt der Friedensprozess den 
Beteiligten tatsächlich eine Art Selbstaufgabe ab, aber nicht in dem befürchteten Sinn: Aufgegeben werden muss nicht die Identität der Großgruppe, verstanden als ihre in ihrem jeweiligen Weg gründende, wandelbare Einzigartigkeit, sondern ihre Opferidentität, also das Besetztsein von bestimmten traumatischen Elementen des kollektiven Geschichtsbildes. Unter dem Einfluss von Opferidentitäten sind Großgruppenbeziehungen nicht mehr frei gestaltbar, sondern unterliegen der Tragik des Wiederholungszwangs. In dem Maße, wie ihr Einfluss zurücktritt, können sich Optionen für friedlichere Koexistenzen, im Kaukasus und anderswo, eröffnen.

\section{Literatur}

Bar On, Dan (2008), The „Others“ Within Us: Constructing Jewish-Israeli Identity, New York: Cambridge University Press.

Burton, John (1990), Conflict: Resolution and Prevention, New York: St. Martins Press.

Coleman, Peter T. (2006), Conflict, Complexity, and Change: A Meta-Framework for Addressing Protracted, Intractable Conflicts - III, Peace and Conflict: Journal of Peace Psychology 12(4), S. 325-348.

De Carvalho, Marco/Klußmann, Jörgen/Rahman, Bahram (2010), Konfliktbearbeitung in Afghanistan. Die Systemische Konflikttransformation im praktischen Einsatz bei einem Großgruppenkonflikt, FES.

Diamond, Louise/McDonald, John (1996), Multi-Track Diplomacy. A Systems Approach to Peace, 3rd ed., Hartford, CT: Kumarian Press.

Fischer, Martina (2006), Civil Society in Conflict Transformation: Ambivalence, Potentials and Challenges, Berghof Research Center for Constructive Conflict Management (http://www. berghof-handbook.net/documents/publications/fischer_cso_ handbook.pdf).

Fisher, Ronald (ed.) (2005), Paving the Way. Contributions of Interactive Conflict Resolution to Peacemaking, Lanham, MD: Lexington Books.

Galtung, Johan/Jacobsen, Carl G./Brand-Jacobsen, Frithjof (2002), Searching for Peace. The Road to Transcend, London, 2nd ed., London: Pluto Press.

Gilovitch, Thomas D. (1981), Seeing the past in the present: the effect of associations to familiar events on judgements and decisions, Journal of Personality and Social Psychology 40(4), S. 797-808.

Gruska, Ulrike (2005), Separatismus in Georgien. Möglichkeiten und Grenzen friedlicher Konfliktregelung am Beispiel Abchasien, Universität Hamburg.

Halbach, Uwe (2010), Ungelöste Regionalkonflikte im Südkaukasus, SWP, Berlin.

Jobelius, Matthias/Krumm, Reinhard (2009), Der KaukasusKonflikt. Russland und Georgien ein Jahr danach, FES, Bonn.
Kaufman, Stuart (2001), Modern Hatreds. The Symbolic Politics of Ethnic War, Ithaca, NY: Cornell University Press.

Ders. (2006), Escaping the Symbolic Politics Trap: Reconciliation Initiatives and Conflict Resolution in Ethnic Wars. Journal of Peace Research 43(2), S. 201-218.

Kaufmann, Walter (2007), Die Rolle von Nichtregierungsorganisationen bei der Bearbeitung von Konflikten im Südkaukasus. In: Klein/Roth (Hg.), NGOs im Spannungsfeld von Krisenprävention und Sicherheitspolitik, Wiesbaden: VS Verlag, S. 299 312 .

Kelman, Herbert (2009), Interactive Conflict Resolution by the Scholar-Practitioner, Zeitschrift für Konfliktmanagement, S. 74-78.

Krell, Gert (2009), Weltbilder und Weltordnungen. Einführung in die Theorie der internationalen Beziehungen, 4. Aufl., Baden-Baden: Nomos.

Lübbe, Anna (1989), Die deutsche Verfassungsgeschichtsschreibung unter dem Einfluss der nationalsozialistischen Machtergreifung. In: Stolleis/Simon (Hg.), Rechtsgeschichte im Nationalsozialismus. Beiträge zur Geschichte einer Disziplin, Tübingen: J.C.B. Mohr, S. 63-78.

Dies. (2007), Ethnopolitische Konflikte: Das Potenzial der Systemaufstellungsmethode. Zeitschrift für Konfliktmanagement 1, S.12-16.

Dies. (2009), Us versus Them: Splitting Dynamics and Turning Points in Ethnopolitical Conflict, Journal of Peace, Conflict and Development 13, http://www.peacestudiesjournal.org. uk/edition/25.

Dies. (2010), Systemic Constellations and their Potential in Peace Work. In: Fitz-Gibbon (Hg.), Positive Peace. Reflections on Peace, Education, Nonviolence and Social Change, Rodopi vibs, S. 49-57.

Meerts, Paul W. (2005), Entrapments in International Negotiations. In: Zartman/Fauré (Hg.), Escalation and Negotiation in International Conflicts, New York: CambridgeUniversity Press, S. 111-140.

Ropers, Norbert (1995), Friedliche Einmischung. Strukturen, Prozesse und Strategien zur konstruktiven Bearbeitung ethnopolitischer Konflikte. Berghof Report No. 1, Berghof Forschungszentrum für konstruktive Konfliktbearbeitung, Berlin.

Ders. (1997), Roles and Functions of Third Parties in the Constructive Management of Ethnopolitical Conflicts. Berghof Arbeitspapier No. 11, Berghof Research Center for Constructive Conflict Management, Berlin.

Ders. (2004), From Resolution to Transformation: The Role of Dialogue Projects. In: Austin et al. (eds.), Transforming Ethnopolitical Conflict. The Berghof Handbook, Berghof Research Center for Constructive Conflict Management, Berlin, S. 225-269.

Sen, Amartya (2007), Die Identitätsfalle. Warum es keinen Krieg der Kulturen gibt, München: C.H.Beck. 
Simon, Fritz B. (2004), Patterns of War. Systemic Aspects of Deadly Conflicts, Heidelberg: Carl Auer.

Ders. (2010), Einführung in die Systemtheorie des Konflikts, Heidelberg: Carl Auer.

Volkan, Vamik (2003), Das Versagen der Diplomatie. Zur Psychoanalyse nationaler, ethnischer und religiöser Konflikte, 3. Aufl., Gießen: Psychosozial Verlag.

Ders. (2004), Das Baum-Modell. In: Geißler (Hg.), Mediation - Theorie und Praxis. Neue Beiträge zur Konfliktregelung, Gießen: Psychosozial Verlag, S. 69-96.

Ders. (2006a), Killing in the Name of Identity. A Study of Bloody Conflicts, Charlottesville, VA: Pitchstone Publishing.

Ders. (2006b), Großgruppen und ihre politischen Führer mit narzisstischer Persönlichkeitsorganisation, in: Kernberg/ Hartmann (Hg.): Narzissmus. Grundlagen - Störungsbilder Therapie, Schattauer, S. 205-227.

Wallach, Tracy (2006), Conflict Transformation: A Group Relations Perspective. In: Fitzduff/Stout (eds.), The Psychology of Resolving Global Conflicts. From War to Peace, Westport: Praeger Publishers, S. 285-305

Wils, Oliver et al. (2006), The Systemic Approach to Conflict Transformation. Concepts and Fields of Application, Berghof Foundation for Peace Support, Berlin.

Wolleh, Oliver (2006), A Difficult Encounter - The Informal Georgian-Abchazian Dialogue Process. Berghof Report No. 12, Berghof Research Center for Constructive Conflict Management, Berlin.

\section{»Ohne Sicherheit ist keine Freiheit«}

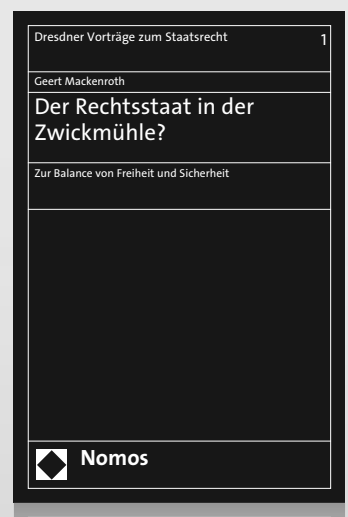

\section{Der Rechtsstaat in der Zwickmühle?}

Zur Balance von Freiheit und Sicherheit

Von Staatsmin a. D. Geert Mackenroth, MdL

2011, 50 S., Rückendraht, 15,-€

ISBN 978-3-8329-6753-6

(Dresdner Vorträge zum Staatsrecht, $B d .1$ )

Der internationale Terrorismus stellt den Staat vor neuartige Herausforderungen. Wie kann der Rechtsstaat die Balance von Freiheit und Sicherheit gewährleisten? Nach der Darstellung der verfassungsrechtlichen Koordinaten erläutert der Autor die Sicherheitsphilosophie des Bundesverfassungsgerichts und analysiert die Schutzpflicht des Staates im Rahmen einer rechtspolitischen Gesamtbetrachtung.

Weitere Informationen: www.nomos-shop.de/13806

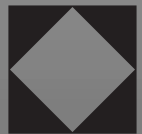

Nomos 